\title{
O efeito de plantas nativas sobre o câncer cervical
}

\section{Gustavo Luiz Padilha}

Instituto Federal de Educação, Ciência e Tecnologia do Rio Grande do Sul (IFRS)

Campus Porto Alegre

(gustavoluizpadilha@yahoo.com.br)

Vitória Garcia La Porta Instituto Federal de Educação, Ciência e Tecnologia do Rio Grande do Sul (IFRS)

Campus Porto Alegre

(vitorialaporta@gmail.com)

Jisette González

Instituto Federal de Educação, Ciência e Tecnologia do Rio Grande do Sul (IFRS) Campus Porto Alegre (jise.glez@gmail.com)

Jordânia dos Santos Pinheiro Instituto Federal de Educação, Ciência e Tecnologia do Rio Grande do Sul (IFRS) Campus Porto Alegre (jordaniaspinheiro@gmail.com)

\section{Gabriel Fernandes Silveira} Instituto Federal de Educação, Ciência e Tecnologia do Rio Grande do Sul (IFRS) Campus Porto Alegre (gabriel.silveira@poa.ifrs.edu.br)

Helana Ortiz Garcia Instituto Federal de Educação, Ciência e Tecnologia do Rio Grande do Sul (IFRS) Campus Porto Alegre (helana.garcia@poa.ifrs.edu.br) 
Bianca Pfaffenseller Hospital de Clínicas de Porto Alegre (HCPA) (bianca.pfaffenseller@gmail.com)

Miriam Anders Apel

Universidade Federal do Rio Grande do Sul (UFRGS) (miriam.apel@ufrgs.br)

\author{
Alessandra Nejar Bruno \\ Instituto Federal de Educação, Ciência e Tecnologia do Rio Grande do Sul (IFRS) \\ Campus Porto Alegre \\ (alessandra.bruno@poa.ifrs.edu.br)
}

\begin{abstract}
Resumo: O potencial de espécies vegetais para a descoberta de novos fármacos antitumorais tem sido reconhecido devido ao uso delas na medicina popular. O câncer cervical possui alta incidência e morbidade, entretanto, os tratamentos convencionais apresentam uma série de limitações. Neste estudo, nós investigamos o efeito do extrato bruto aquoso obtido de folhas de uma espécie da família Myrtaceae (EBA), um óleo essencial das folhas desta mesma espécie (OE1) e óleo essencial extraído de flores de uma espécie da família Asteraceae (OE2) em células de uma linhagem humana de câncer cervical ( $\mathrm{SiHa}$ ) e em uma linhagem não tumoral de queratinócitos humanos (HaCaT). As células foram mantidas em condições padrão e tratadas por $24 \mathrm{~h}$ com diferentes concentrações dos tratamentos e então submetidas ao ensaio de MTT $(0,5 \mathrm{mg} / \mathrm{ml})$. Os três tratamentos utilizados nas diferentes concentrações induziram inibição significativa na viabilidade das células tumorais a partir de concentrações baixas, entretanto, o IC50 de um tratamento que induziu um efeito pronunciado nas células tumorais, não causou tamanha inibição na viabilidade da linhagem não tumoral. O potencial terapêutico dos tratamentos utilizados enfatiza a importância de investimentos na continuidade destes estudos e projetos relacionados à investigação de espécies vegetais como aliados no combate ao câncer.
\end{abstract}

Palavras-chave: Plantas nativas; Câncer cervical; Viabilidade celular.

\title{
The effect of native plants on cervical cancer
}

Abstract: The potential of plant species for the discovery of new antitumor drugs has been recognized due to their use in folk medicine. Cervical cancer has high incidence and morbidity; however, conventional treatments have a number of limitations. In this study, we investigated the effect of the crude aqueous extract obtained from leaves of a species of the family Myrtaceae (EBA), an essential oil of leaves of this species (OE1) and essential oil from flowers of a species of the family Asteraceae (OE2) on human cervical cancer cells (SiHa line) and on a non-tumor human keratinocyte line (HaCaT). Cells were maintained under standard conditions and treated for 24 hours at different concentrations of the treatments and then submitted to the MTT assay $(0.5 \mathrm{mg} / \mathrm{mL})$. The three treatments used induced significant inhibition in the viability of tumor cells from low concentrations, however, the IC50 of a treatment that induced a pronounced effect on tumor cells did not cause such 
inhibition in the viability of the non-tumor line. The therapeutic potential of the treatments used emphasizes the importance of investments for continuity of these studies and for other projects related to the investigation of plant species as allies in the fight against cancer.

Keywords: Native plants; Cervical cancer; Cell viability.

\section{INTRODUÇÃO}

O câncer é um problema de saúde pública mundial, constituindo a segunda causa de morte por enfermidades no Brasil. Nas últimas décadas, o número de novos casos no registro brasileiro de câncer tem crescido, ressaltando a importância da doença e seu impacto social e econômico (INCA, 2018). Era estimado para 2018, o surgimento de 18,1 milhões de novos casos de câncer (BRAY et.al., 2018), além de representar $13 \%$ de todas as causas de morte do mundo (GUERRA e GALLO, 2005).

Câncer é definido como um conjunto de doenças caracterizadas pelo crescimento anormal, progressivo e desordenado de células (ALMEIDA et al., 2005). Alterações nos processos normais de crescimento, proliferação e morte celular estão associadas à mutações que ocorrem preferencialmente em genes supressores de tumores e/ou de protooncogenes. Diferentes tipos de câncer também têm sido associados com alterações em genes associados ao reparo de DNA (TENEN, 2003).

Nosso grupo de pesquisa vem realizando ensaios biológicos utilizando culturas de células de tumores femininos, com foco, principalmente, no câncer de colo uterino humano. O câncer cervical permanece como uma doença de alta prevalência, incidência e mortalidade, sendo o quarto tipo de câncer mais comum em mulheres, e o sétimo na classificação geral (PARKIN e BRAY, 2006). Em países em desenvolvimento, esta patologia tem mantido altos níveis de incidência, sendo o terceiro tipo de câncer com maior estimativa de novos casos entre as mulheres no Brasil (INCA, 2018) Sabe-se que a infecção pelo papilomavírus humano (HPV) associada a outros fatores de risco como o histórico de outras doenças sexualmente transmissíveis, tabagismo e uso de contraceptivo oral, representam um importante papel na progressão das lesões escamosas intra-epiteliais para a malignidade em mulheres (WALBOOMERS et al., 1999; LIU et al., 2000). Infelizmente alguns estudos revelaram recorrência de câncer de colo uterino após histerectomia radical em cerca de $20 \%$ dos pacientes (YUAN et al., 1999), bem como uma alta taxa de 
recorrência para o câncer cervical invasivo mesmo após o tratamento da neoplasia intra-epitelial cervical (SOUTTER et al., 2006). Além disso, tratamentos convencionais como a quimioterapia e a radioterapia, não atuam exclusivamente sobre as células tumorais. Esta toxicidade indiscriminada é a causa dos inúmeros efeitos adversos observados e a consequente influência na qualidade de vida dos pacientes.

Por estas razões, muitos estudos baseados em princípios ativos de plantas têm investigado a atividade antiproliferativa ou citotóxica em células tumorais visando a geração de novos agentes antineoplásicos. Neste contexto é importante destacar a biodiversidade brasileira como uma fonte extraordinária de espécies vegetais com diferentes moléculas ativas que possuem potenciais efeitos biológicos e terapêuticos para doenças como o câncer.

Substâncias orgânicas originadas de fontes naturais há muito tempo são utilizadas no tratamento de inúmeras doenças na chamada medicina popular. Grande parte destas substâncias, quando levadas ao laboratório, demonstraram propriedades químicas e biológicas promissoras e de grande interesse biotecnológico. A análise da origem dos fármacos encontrados no mercado revela a importância do desenvolvimento de novos medicamentos baseados em ativos vegetais, já que mais da metade dos novos medicamentos lançados a partir de 1981 são derivados de produtos naturais (CHIN et al., 2006; NEWMAN e CRAGG, 2016).

Alguns exemplos de medicamentos de origem vegetal e com atividade antitumoral são a Vimblastina e a vincristina, isolados das folhas da espécie Catharanthus roseus (L.) G. Don. (Apocynaceae), popularmente conhecida como vinca. Estas espécies vegetais eram originalmente utilizadas no tratamento de diabetes e posteriormente foram descritos com atividade antimitótica e aprovadas pelo FDA (Food and Drug Administration) como agentes anticancerígenos (NEWMAN et al., 2000). Os quimioterápicos etoposídeo e o teniposídeo foram obtidos a partir das espécies $P$. peltatum e $P$. emodii, tradicionalmente utilizadas pelas populações nativas da América e da Ásia no tratamento do câncer de pele e verrugas (BRANDÃO et al., 2010). Outros exemplos importantes incluem o paclitaxel (Taxol®), um diterpeno da família dos taxanos extraído a partir de extratos de casca da árvore de Taxus brevifolia Nutt. (Taxaceae) (ALTMANN e GERTSCH., 2007), bem como o irinotecan e o topotecano, obtidos a partir de uma árvore ornamental 
chinesa conhecida como Camptotheca acuminata Decne (Cornaceae) (BRANDÃO et al., 2010).

O nosso grupo de pesquisa tem demonstrado os efeitos significativos de diferentes preparações oriundas de plantas nativas sobre a viabilidade de células tumorais, bem como sua atividade em outros parâmetros biológicos importantes para a sobrevivência, crescimento e invasão das mesmas. Uma das espécies testadas é representante da família Asteraceae. Esta é a maior família de angiospermas, compreendendo 25.000 espécies pertencentes a 1.600 gêneros (BREMER, 1994). No Brasil, a família está representada por aproximadamente 196 gêneros e cerca de 1.900 espécies (BARROSO et al., 1991). Membros desta família são amplamente utilizadas na medicina popular e estudadas devido a sua atividade biológica e composição química, com destaque a abundância de flavonóides e cientificamente descritas com atividades antibacteriana, antifúngica, larvicida, inseticida, antiparasitária, nematicida, anti-hiperglicêmica e antioxidante (ANDREOTTI et al., 2013; ROMAGNOLI et al., 2005; SCHIAVON et al., 2015). A exemplo das propriedades dessa família, é reconhecido a Helianthus annuus $L$ no efeito contra a Epilepsia; como calmante tem-se Matricaria recutita L; no combate a tosse e queilite angular é indicado a espécie Sonchus oleraceus L (SILVA e ANDRADE, 2013). Além disso, possuem alta disponibilidade já que apresentam alta taxa de germinação e produzem flores e sementes o ano inteiro (BARROSO et al., 1991). Representantes desta família são conhecidamente produtoras de óleos essenciais com importância comercial já descrita (ANDREOTTI et al., 2013). Óleos essenciais são compostos de baixo peso molecular oriundos do metabolismo secundário de plantas e de composição química caracterizada por atividades farmacológicas de grande interesse biotecnológico (ADAMS, 2009).

Outra família amplamente estudada em nosso laboratório, abrange membros pertencentes à família Myrtaceae. As mirtáceas destacam-se por suas propriedades medicinais, já que seus extratos apresentam efetivos antihipertensivos (Eugenia uniflora), hipoglicemiantes (Eugenia jambola), anti-bacteriana (Calyptranthes lucida), antifúngicos (Pimenta dioica), antioxidantes (Eugenia involucrata) e anti inflamatório (Psidium cattleyanum), (CONSOLINI e SARUBBIO, 2002; LIMA et al., 2002; BRUNETTI et al., 2006; STEFANELLO et al., 2011). Devido a estas propriedades, 
membros desta família são conhecidos por seu potencial para a obtenção de fármacos (SILVA, 2006).

Considerando a importância de estudos que envolvam produtos naturais, a riqueza de substâncias ativas presentes em plantas nativas e o potencial destas substâncias como potenciais agentes antineoplásicos, este artigo descreve alguns resultados de nossa pesquisa, demonstrando a influência do tratamento com algumas preparações oriundas de espécies vegetais nativas sobre a viabilidade de células humanas de câncer de colo de útero. As espécies estudadas neste artigo não serão declaradas devido à intenção de patente.

\section{METODOLOGIA}

Linhagens celulares: As linhagens de origem neoplásica escamosa da cérvice uterina humana ( $\mathrm{SiHa}$ ) e a linhagem não tumoral imortalizada de queratinócitos humanos (Hacat) foram obtidas da "American Type Culture Collection" (ATCC, USA). As culturas foram mantidas em meio de cultura DMEM (Dulbecco's Modified Eagle's Medium) suplementado com soro fetal bovino $10 \%$ e mantidas em estufa a $37^{\circ} \mathrm{C}$ em atmosfera com $5 \%$ de $\mathrm{CO}_{2}$.

Extrato bruto aquoso: A coleta das folhas de uma espécie da família Myrtaceae foi realizada em abril de 2017, no Município de Porto Alegre-RS. As folhas foram lavadas e secas em estufa a $60^{\circ} \mathrm{C}$ por 4 dias. Posteriormente, estas foram trituradas e o pó obtido foi dissolvido em meio de cultura DMEM (não suplementado). A solução obtida (EBA) foi filtrada em membrana com poros de 0,22 $\mu \mathrm{m}$.

Óleos essenciais: Após identificação taxonômica, folhas de uma espécie de Myrtaceae (OE1) e flores de uma espécie vegetal da família Asteraceae (OE2) foram coletadas em áreas de ocorrência natural no Rio Grande do Sul e submetidas ao processo de hidrodestilação, separadamente, em aparelho do tipo Clevenger durante 3 horas. A identificação química dos óleos essenciais foi realizada por cromatografia a gás acoplado a espectrômetro de massas (GC-MS) equipado com DB-5 ligado a uma coluna capilar de sílica (30 $\mathrm{m} \times 0,25 \mathrm{~mm} \times 0,25 \mathrm{~mm}$ ), sendo os componentes do óleo identificados por comparação entre seu índice de retenção e seus espectros de massa. Os dados de referência foram obtidos a partir da literatura 
e base de dados NIST 62. Para a realização dos ensaios com as culturas celulares, preparou-se uma solução mãe pela solubilização dos óleos no veículo propilenoglicol em diferentes proporções - 1:5 para a espécie da família Asteraceae (OE2) e 1:10 para a espécie da família Myrtaceae (OE1).

Tratamentos: Para utilização dos óleos essenciais, é necessário a solubilização dos mesmo em veículos, levando em conta a análise qualitativa visual das soluções e o limite de toxicidade da substância veículo constatada na literatura. Ambos, OE1 e OE2, foram solubilizados no veículo propilenoglicol, sendo o OE1 solubilizado na proporção de 1:10 e o OE2 na proporção de 1:5. Em todos os ensaios, poços controles (apenas meio de cultura DMEM) e poços controle veículo (meio de cultura DMEM e propilenoglicol na concentração correspondente) foram realizados. Para o EBA foi realizado apenas o controle com meio DMEM, já que este não necessita solubilização em veículo.

Viabilidade celular: A viabilidade das células após os respectivos tratamentos foi verificada através do ensaio com MTT \{brometo de [3-(4,5dimetiltiazol-2-yl)-2-5-difeniltetrazólio]\}. Este consiste na absorção do sal MTT pelas células, sendo reduzido no interior da mitocôndria a um produto chamado Formazan. As células foram plaqueadas na densidade de $2,8 \times 10^{3}$ células por poço em uma placa de 96 poços. Após adesão, as células foram tratadas com diferentes concentrações dos óleos OE1 na faixa entre $(0,5 \mu \mathrm{g} / \mathrm{mL}$ até $30 \mu \mathrm{g} / \mathrm{mL}$ ), OE2 (de $0,01 \mu \mathrm{g} / \mathrm{mL}$ até $30 \mu \mathrm{g} / \mathrm{mL}$ ) em triplicata e com o extrato bruto aquoso EBA (de $0,5 \mathrm{mg} / \mathrm{mL}$ até $20 \mathrm{mg} / \mathrm{mL}$ ) em duplicata durante 24 horas. Ao término do tratamento, o conteúdo dos poços foi descartado para adição de MTT (0,5 mg/mL). Após 3,5 horas de incubação, os cristais de formazona foram solubilizados em dimetisulfóxido (DMSO) e foi realizada a leitura da absorbância a $545 \mathrm{~nm}$ e $630 \mathrm{~nm}$ em leitor de placas. Utilizando os resultados desse ensaio foi possível calcular a concentração inibitória média (IC50) através da construção dos gráficos no software GraphPad Prism 5 para a obtenção de uma linha de tendência linear e cálculo do IC50.

Após esses resultados, foi realizado um ensaio para verificar somente o efeito do tratamento OE2 durante 24, 48 e 72 horas de tratamento sobre as células da linhagem não tumoral Hacat. Para isso, foi utilizado apenas a concentração de IC50 deste óleo obtida na linhagem tumoral como tratamento, e a análise se deu 
utilizando a mesma metodologia descrita para verificar o efeito dos tratamentos na viabilidade das células tumorais.

Análise Estatística: Com base nos resultados obtidos através de pelo menos três experimentos independentes, realizados em duplicata, calculou-se as médias e os desvios padrões das diferentes análises. Para a comparação entre os grupos, foi aplicada análise de variância de uma via (ANOVA) seguido de teste de Tuckey para comparações múltiplas. Foram analisadas as diferenças entre cada concentração e o controle de meio DMEM considerado significativas quando a análise estatística apresentar $p<0,05$.

Questões Éticas e biossegurança: O trabalho com linhagens celulares exclui a necessidade de utilização de animais para a pesquisa e, portanto, exclui a possibilidade de questionamentos éticos. Além disto, todas as normas de biossegurança para armazenamento e utilização das mesmas, já foram implementadas em nosso laboratório e estão sendo rigorosamente cumpridas. $O$ descarte dos resíduos químicos e biológicos gerados seguiu o procedimento padrão usual já estabelecido nos laboratórios do curso técnico de Biotecnologia do IFRSPOA.

\section{RESULTADOS}

\section{Extrato Bruto aquoso (EBA)}

O tratamento com o extrato bruto aquoso (EBA) obtido a partir de folhas de um representante da família Myrtaceae, foi capaz de inibir entre $18 \%$ e $52 \%$, a viabilidade das células tumorais SiHa. A concentração capaz de promover uma maior inibição em relação do controle foi $20 \mathrm{mg} / \mathrm{mL}$ (Figura 1). A partir do teste com concentrações de 0,5 a $20 \mathrm{mg} / \mathrm{mL}$ foi possível estabelecer também o IC50 de 7,8 $\mathrm{mg} / \mathrm{mL}$ nas células de SiHa após 24 horas de tratamento. 
Figura 1 - Efeito de diferentes concentrações de EBA sobre a viabilidade da linhagem SiHa 24h. Os dados mostram a média e o desvio padrão de três experimentos independentes, realizados em duplicatas. ${ }^{*} \mathrm{P}<0,05$ (ANOVA de uma via seguido pelo teste de Tukey).

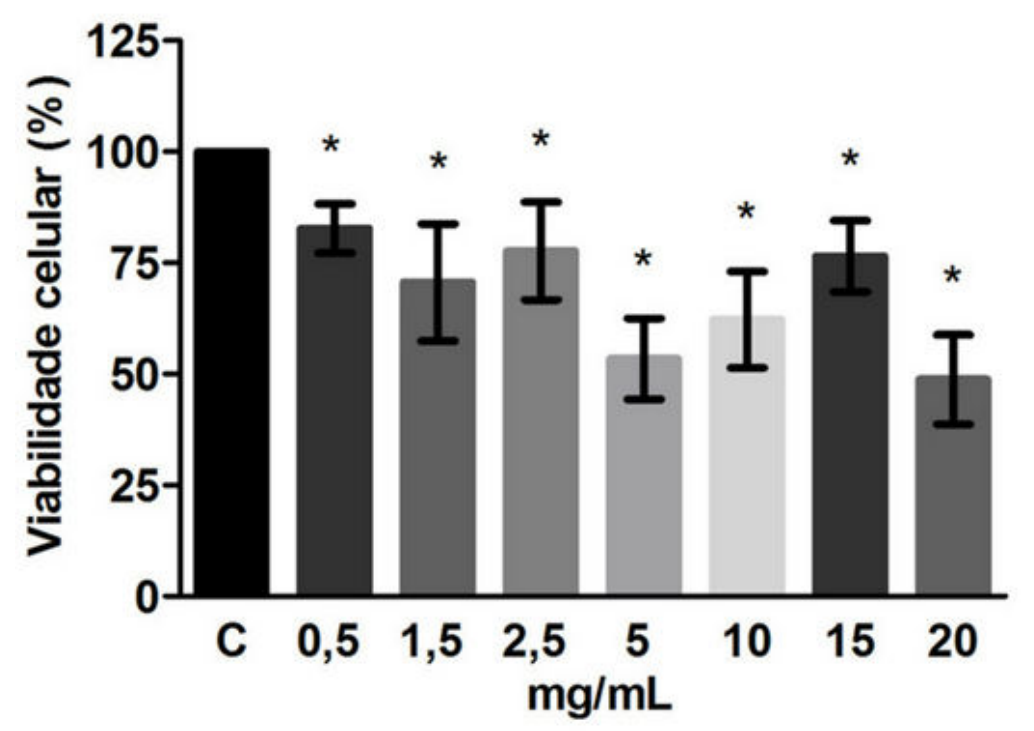

\section{Óleo essencial Myrtaceae (OE 1)}

O tratamento com o óleo essencial extraído das folhas de uma planta nativa da família Myrtaceae (OE1) induziu um efeito significativo de diminuição da viabilidade das células tumorais testadas entre $49 \%$ e $81 \%$ (Figura 2). A concentração que apresentou um efeito inibitório maior em relação ao controle foi $0,5 \mu \mathrm{g} / \mathrm{mL}$. Além disso, o veículo utilizado não induziu uma diminuição significativa na viabilidade das células testadas em concentrações menores que $1,5 \mu \mathrm{g} / \mathrm{mL}$ (Figura 2). A partir destes resultados foi possível encontrar um IC50 de 2,1 $\mu \mathrm{g} / \mathrm{mL}$ para o OE1 nas células SiHa. 
Figura 2 - Efeito de diferentes concentrações de OE1 sobre a viabilidade da linhagem SiHa $24 \mathrm{~h}$. Os dados mostram a média e o desvio padrão de três experimentos independentes, realizados em triplicatas. ${ }^{*} \mathrm{P}<0,05$ (ANOVA de uma via seguido pelo teste de Tukey).

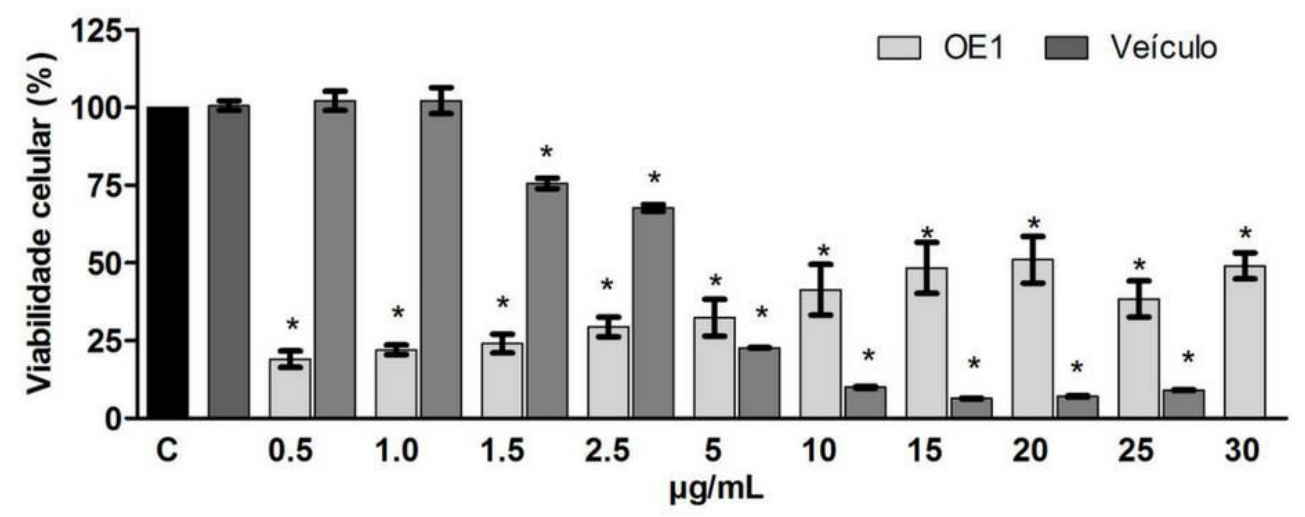

\section{Óleo essencial Asteraceae (OE2)}

O tratamento com óleo essencial extraído das flores de uma espécie vegetal nativa da família Asteraceae (OE2) também foi capaz de inibir de forma significativa a viabilidade das células tumorais (30\% até 94\%) (Figura 3). Como podemos observar ainda na Figura 3, o efeito mais proeminente na inibição da viabilidade das células tumorais em relação do controle se deu na concentração $0,5 \mu \mathrm{g} / \mathrm{mL}$. $O$ cálculo do IC50 para OE2 nas células SiHa em 24 horas de tratamento revelou um valor de 0,072 $\mu \mathrm{g} / \mathrm{mL}$. Em adição, nós também testamos o IC50 de OE2 nas células não tumorais HaCaT durante 24, 48 e 72 horas. Este ensaio revelou que o tratamento com IC50 das células tumorais alterou a viabilidade das células não tumorais em $42 \%$ em 24 horas de tratamento, 19\% em 48 horas e 21\% em 72 horas de tratamento, demonstrando um efeito distinto de OE2 para células tumorais e não tumorais. Além disto, o veículo utilizado não apresentou efeito inibitório na viabilidade das células de HaCat, viabilizando a metodologia e portanto, os resultados com os óleos essenciais testados (Figura 4). 
Figura 3 - Efeito de OE2 em diferentes concentrações sobre a viabilidade da linhagem SiHa durante $24 \mathrm{~h}$ de tratamento. Os dados mostram a média e o desvio padrão de três experimentos independentes, realizados em triplicata * $\mathrm{P}<0,05$ (ANOVA de uma via seguido pelo teste de Tukey).

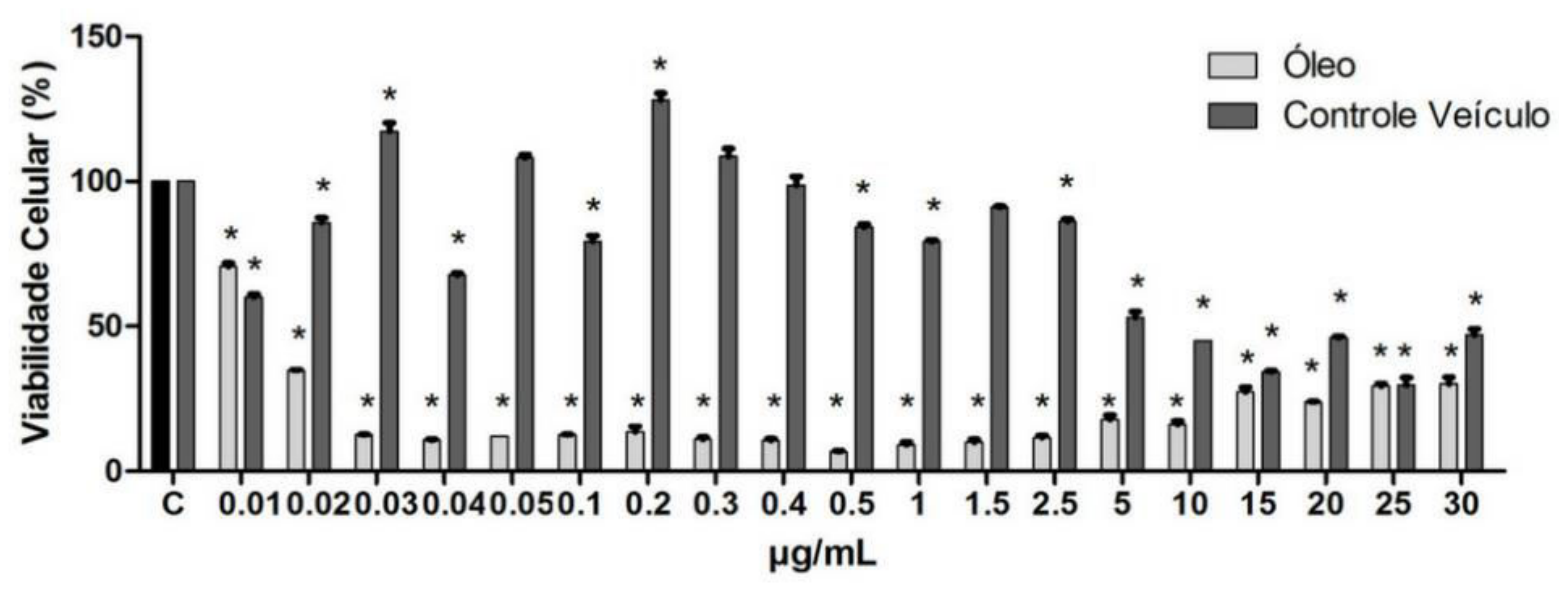

Figura 4 - Efeito de OE2 na concentração IC50 $(0,072 \mu \mathrm{g} / \mathrm{mL})$ sobre a viabilidade da linhagem HaCaT durante 24, 48 e 72 horas de tratamento. Os dados mostram a média e o desvio padrão de três experimentos independentes, realizados em triplicata * $\mathrm{P}<0,05$ (ANOVA de uma via seguido pelo teste de Tukey).

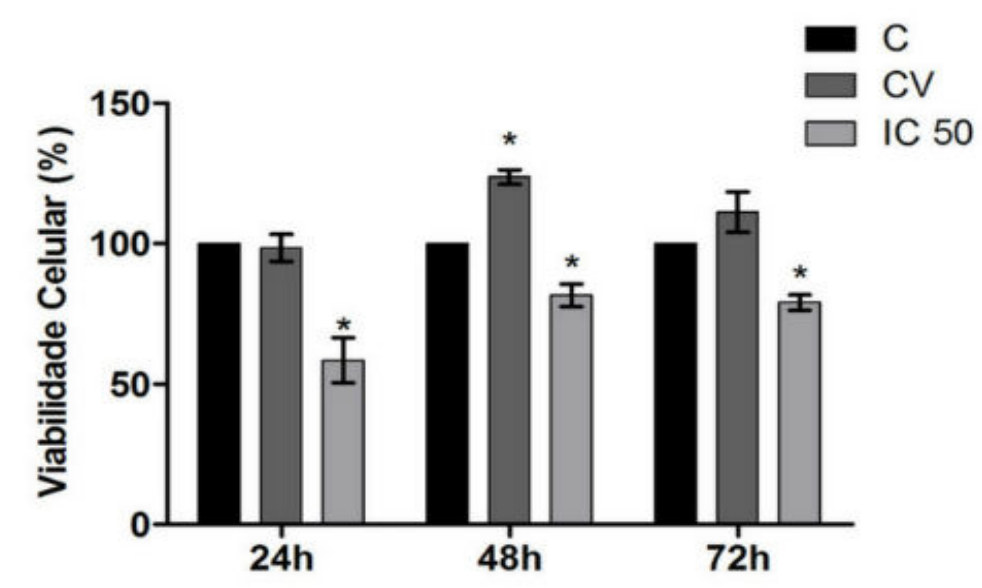

\section{DISCUSSÃO E CONCLUSÃO}

A história dos medicamentos está intimamente relacionada aos produtos naturais, sendo que mais de $60 \%$ dos medicamentos antitumorais disponíveis na 
clínica apresentam origem relacionada a alguma fonte natural (NEWMAN e CRAGG, 2016). Apesar da introdução de novos fármacos no arsenal terapêutico do câncer, muitos tumores ainda não dispõem de tratamento eficiente. Por outro lado, fontes naturais ainda estão disponíveis em abundância e oferecem substâncias de interesse terapêutico. Por estes motivos, nosso grupo de pesquisa tem focado esforços na obtenção, estudo e triagem de produtos de origem vegetal com atividades antitumorais promissoras.

Os resultados demonstrados neste artigo indicam um potencial efeito antineoplásico dos três tratamentos testados, já que as diferentes concentrações dos mesmos foram capazes de inibir de forma significava a viabilidade das células tumorais utilizadas. Foi observado um efeito significativo de inibição da viabilidade celular com o IC50 do OE1 e OE2, respectivamente, nas concentrações de 2,1 $\mu \mathrm{g} / \mathrm{mL}$ e $0,072 \mu \mathrm{g} / \mathrm{mL}$ com redução de células viáveis entre $40 \%$ e $88 \%$ para o OE1 e de $42 \%$ até $88 \%$ para o OE2. Estas concentrações são consideradas baixas quando comparadas ao valor encontrado para o fármaco padrão cisplatina, que por sua vez, é de $24.10 \mu \mathrm{g} / \mathrm{mL}$ (NG et al., 2011). Neste sentido, é importante destacar que os valores de IC50 para drogas com atividade antitumoral situam-se entre $6 \mu \mathrm{M}$ e $16 \mu \mathrm{M}$ (RABELO et al., 2002). Estes dados, enfatizam o potencial terapêutico dos tratamentos aqui descritos, já que drogas efetivas em concentrações baixas estão relacionadas com a indução de menos efeitos adversos.

Foi observado também, que em concentrações mais baixas, o tratamento com OE1 foi capaz de induzir uma maior inibição na viabilidade das células tumorais. Entretanto, nessas concentrações, o veículo utilizado também exerceu efeitos significativos na mesma, o que nos leva a focar em concentrações menores deste tratamento, já que são mais efetivas e sem influência do veículo. Muitas vezes concentrações maiores ou um maior tempo de exposição ao tratamento não refletem em efeitos mais pronunciados em parâmetros celulares analisados in vitro. Efeitos clássicos como o padrão de dose dependência podem não ocorrer, refletindo a indução de diferentes mecanismos referentes à sinalização celular e/ou de uma absorção menos eficiente, muitas vezes observada em estudos com óleos essenciais. Em relação ao tempo de exposição ao tratamento, o mecanismo de tolerância ou resistência a drogas, pode justificar o menor efeito de OE2 em 48 horas de tratamento em relação à exposição de 24 horas. Já foi demonstrado que os 
tumores podem apresentar heterogeneidade molecular, o que lhes confere diferente sensibilidade e vias de resposta aos tratamentos. A resistência pode ocorrer por alterações nas células induzidas pelo tratamento (resistência extrínseca), ou pela seleção de subpopulações celulares com maiores limiares aos danos induzidos pelo tratamento (resistência intrínseca) (SANDERS et al., 2012).

O investimento na busca de novos fármacos antineoplásicos é de grande relevância, já que os tratamentos convencionais atualmente disponíveis, afetam de forma indiscriminada as células normais, provocando efeitos adversos, o que pode ser observado, por exemplo na terapia com as antraciclinas provocando efeitos como estomatite, náuseas, alopecia entre outros (HORTOBÁGYI, 1997). No caso do câncer cervical, a intervenção cirúrgica, além de invasiva, não exclui a possibilidade de recorrência do tumor (SOUTTER et al., 2006). A capacidade terapêutica da radioterapia no caso de câncer de colo do útero localmente avançado é limitada pelo tamanho do tumor, uma vez que as doses necessárias para tratar grandes tumores excedem o limite de toxicidade em tecido normal (FLETCHER, 1973). Em relação à quimioradioterapia convencional, também foi demonstrado um prognóstico limitado, com taxas de sobrevivência de 5 anos em aproximadamente 60\% dos casos (MORRIS et al., 1999; EIFEL et al., 2004). Além disso, a natureza não seletiva da quimioterapia e da radioterapia leva a efeitos secundários graves em células normais com um elevado índice de proliferação. A cisplatina é um potente agente quimioterápico amplamente utilizado no tratamento do câncer, entretanto, é frequentemente associada à nefrotoxicidade e ototoxicidade, entre outros efeitos adversos (JONES e CHESNEY, 1995; HYPPOLITO et al., 2005). Estes efeitos limitam assim a dose eficaz de fármaco anticancerígeno que pode ser administrada e os efeitos adversos induzidos têm um impacto significativo na qualidade de vida dos pacientes (EIFEL et al., 2004). Estes dados justificam o interesse crescente no desenvolvimento de novos produtos farmacêuticos e estratégias alternativas contra o câncer. Em nosso estudo, nós verificamos que um dos tratamentos (OE2) que induziu um efeito de grande proporção na viabilidade das células tumorais, não foi capaz de induzir o mesmo efeito nas células não tumorais (Figura 4) durante 24 horas de tratamento, demonstrando um efeito distinto em células tumorais e não tumorais. O efeito aqui descrito se manteve quando as células não tumorais foram submetidas a este tratamento também nos tempos de 48 e 72 horas. 
Além disso, para a pesquisa envolvendo produtos naturais, a parte utilizada da planta, bem como a forma no qual as soluções são preparadas, são fatores diferenciais para a extração das moléculas de interesse. Sabe-se que entre as pesquisas envolvendo extratos e óleos essenciais de plantas, se destacam a presença de compostos polifenólicos, fenólicos e terpênicos, como responsáveis potenciais por diferentes efeitos biológicos (BAKKALLI et al., 2008; VICTORIA, 2013). Desta forma, em nosso laboratório, temos estudado as propriedades biológicas de diferentes plantas nativas em suas diferentes apresentações. Estudos dos aspectos fitoquímicos e farmacológicos de espécies de plantas nativas demonstram a presença de moléculas, como esteróis e triterpenos, conhecidos por seus efeitos antioxidantes, antinflamatórios, analgésicos e antimicrobianos (PARENTE et al., 2002). Um resultado normalmente requerido em estudos envolvendo espécies vegetais, é a identificação química e isolamento dos compostos majoritários presentes nos tratamentos testados, visando a análise da contribuição isolada de cada composto para indução dos efeitos observados. Entretanto, no presente estudo, não podemos descartar a possibilidade de uma ação sinérgica das moléculas constituintes de tais espécies vegetais para a contribuição dos efeitos aqui demonstrados.

Os estudos de viabilidade celular em culturas celulares utilizando diferentes concentrações de uma droga em teste e a obtenção do IC50, como demonstrado neste estudo, são de grande relevância para a triagem das drogas com real potencial terapêutico. Entretanto, dada a confirmação de efeitos inibitórios sobre a viabilidade de células tumorais, é importante assegurar a continuidade de testes que analisem o potencial antineoplásicos das drogas testadas através de outras metodologias. Um dos parâmetros importantes e que também realizados em nosso laboratório, é a investigação dos mecanismos de morte celular induzido por um determinado tratamento, caso seja observado um efeito de citotoxicidade. Alterações na coordenação dos diferentes tipos de morte celular (apoptose, autofagia, necrose, mitose catastrófica e senescência) estão intimamente relacionadas à formação de tumores, assim como, aos efeitos adversos induzidos por diferentes drogas citotóxicas. A demonstração de que a apoptose é um modo inato de defesa antineoplásica e que vários agentes quimioterápicos agem através da indução desse tipo de morte celular, tornou a investigação dos mecanismos de morte celular uma 
prática importante na busca por novos tratamentos antitumorais quando estes demonstram efeitos promissores nos estudos de triagem de viabilidade celular (GRIVICICH et al., 2007).

Em conclusão, os resultados descritos através deste artigo são promissores como passos iniciais na busca por novas alternativas terapêuticas para o câncer do colo do útero. Entretanto, são necessários estudos adicionais para determinar quais as moléculas estão de fato contribuindo para os efeitos observados, assim como a investigação de outros parâmetros biológicos relevantes para a manutenção e expansão de células tumorais, tais como processos de migração, adesão celular, capacidade de formação de colônias, entre outros processos já padronizados por nosso grupo de pesquisa. De qualquer forma, os resultados aqui demonstrados são exemplos que evidenciam a relevância de investimentos em pesquisas abrangendo diferentes preparações com ativos vegetais para o desenvolvimento de novas alternativas terapêuticas para o câncer.

\section{REFERÊNCIAS}

ADAMS, R. P. Identification of Essential Oil Components by Gas Chromatography/Mass Spectrometry. Journal of the American Society for Mass Spectrometry, v. 16, n. 11, p. 1902-1903, 2009.

ALMEIDA, V. L., LEITÃO, A., REINA, L.C. B., MONTANARI, C.A., DONNICI, C.L., LOPEZ, M.T.P. Câncer e agentes antineoplásicos ciclo-celular específicos e ciclo-celular não específicos que interagem com o DNA: uma introdução. Química Nova. v. 28, n. 1, p. 118-129, 2005.

ALTMANN, K. H., GERTSCH, J. Anticancer drugs from nature - natural products as a unique source of new microtubule-stabilizing agents. Natural Product Reports. v. 24, n. 2, p. 327-357, 2007.

ANDREOTTI, R., GARCIA, M. V., CUNHA, R. C., BARROS, J. C. Protective action of Tagetes minuta (Asteraceae) essential oil in the control of Rhipicephalus microplus Canestrini,1887) (Acari: Ixodidae) in a cattle pen trial. Veterinary Parasitology, v. 197, p. 341-345, 2013.

BAKKALLI, F., AVERBECK, S., AVERBECK, D., IDAOMAR, M. Biological effects of essential oils: a review. Food and Chemical Toxicology, v. 46, p. $446-475$, 2008. 
BARROSO, G. M., PEIXOTO, A. L., ICHASO, C. L. F., COSTA, C. G., GUIMARÃES, E. F., LIMA, H. C. Sistemática de Angiospermas do Brasil. Viçosa: Universidade Federal de Viçosa, Imprensa Universitária, v. 3, p. 237-314, 1991.

BRANDÃO, H.N., DAVID, J.P., COUTO, R.D., NASCIMENTO, J. A. P., DAVID, J.M.Chemistry and pharmacology of antineoplasic chemoterapeutical derivatives from plants.Química Nova, São Paulo ,vol.33, n. 6, 2010.

BRAY, F., FERLAY, J., SOERJOMATARAM, I., SIEGEL, R. L., TORRE, L. A., JEMAL, A. Global cancer statistics 2018: GLOBOCAN estimates of incidence and mortality worldwide for 36 cancers in 185 countries. American Cancer Society.Cancer Journal for Clinicians, v. 68, n.6, p. 394-424, 2018.

BREMER, K. Asteraceae: Cladistics and classification. Portland: Timber Press, p. 429, 1994.

BRUNETTI, I., VENDRAMINI, R., JANÚARIO, A. Effects and Toxicity of Eugenia punicifolia extracts in Streptozotocin-Diabetic Rats. Pharmaceutical, v. 44, n. 1, p. 35-43, 2006.

CHIN, Y. W., BALUNAS, M. J., CHAI, H. B., KINGHORN, A. D. Drug discovery from natural sources. The AAPS J. v. 8, n. 2, p. E239- 53, 2006.

CONSOLINI, A., SARUBBIO, M. Pharmacological effects of Eugenia uniflora (Myrtaceae) aqueous crude extract on rat's heart. Journal of Ethnopharmacology, v. 8, n. 1 , p. $57-63,2002$.

EIFEL, P. J., WINTER, K., MORRIS, M., LEVENBACK, C., GRIGSBY, P. W., COOPER, J., ROTMAN, M., GERSHENSON, D., MUTCH, D. G. Pelvic irradiation with concurrent chemotherapy versus pelvic and para-aortic irradiation for high-risk cervical cancer: An update of Radiation Therapy Oncology Group Trial (RTOG) 90-01, v. 22, n. 5, p. 872-880, 2004.

FLETCHER, G. Textbook of Radiotherapy. $2^{\mathrm{a}}$ ed. Philadelphia: Lea and Febiger, p. 816,1973 .

GRIVICICH, I., REGNER, A., ROCHA, A. B. Morte Celular por Apoptose. Revista Brasileira de Cancerologia, v. 53, n. 5, p. 335-343, 2007.

GUERRA, M. R., GALLO, C. V. M. Risco de câncer no Brasil: tendências e estudos epidemiológicos mais recentes. Revista brasileira, v. 51, n. 3, p. 227234, 2005.

HORTOBÁGYI, G.N. Antraciclinas no Tratamento do Câncer. Drugs, v. 54, n. 4, p. 1-7, 1997.

HYPPOLITO, M. A., OLIVEIRA, J. A. A., LESSA, R. M., ROSSATO, M. Otoproteção da amifostina aos efeitos ototóxicos da cisplatina: estudo em cobaias albinas por emissões otoacústicas produtos de distorção e microscopia eletrônica de 
varredura. Revista Brasileira de Otorrinolaringologia, v.71, n. 3, p. 268-73, 2005.

INSTITUTO NACIONAL DE CÂNCER JOSÉ ALENCAR GOMES DA SILVA (INCA). Estimativa 2018: Incidência de Câncer no Brasil. Rio de Janeiro, p.1-129, 2017.

JONES, D., CHESNEY, R. Renal toxicity of cancer chemotherapeutic agents in children: ifosfamide and cisplatin. Current opinion in pediatrics, v. 7, p. 208-213, 1995.

LIMA, V.L.A.G., MÉLO, E.A., LIMA, D.E.S. Fenólicos e carotenóides totais em pitanga. Scientia Agricola, v. 59, n. 3, p. 447-450, 2002.

LIU, Y., MCKALIP, A., HERMAN B. Human papillomavirus type 16 E6 and HPV-16 E6/E7 sensitize human keratinocytes to apoptosis induced by chemotherapeutic agents: roles of p53 and caspase activation. Journal of Cellular Biochemistry, v. 78, p. 334-49, 2000.

MORRIS, M., EIFEL, P., LU, J., GRIGSBY, P. Pelvic radiation with concurrent chemotherapy compared with pelvic and para-aortic radiation for high-risk cervical cancer. The new England Journal of Medicine, v. 340, p. 1137-1143, 1999.

NEWMAN, D. J., CRAGG, G. M. Natural products as sources of new drugs from 1981 to 2014. J. Journal of Natural Products, v. 79, n. 3, p. 629-661, 2016.

NEWMAN, D.J., CRAGG, G.M., SNADER, K.M. The influence of natural products upon drug discovery. Journal of Natural Products; v. 17, p. 215-234, 2000.

NG, W. K., YAZAN, L.S., MAZNAH, I. Thymoquinone from Nigella sativa was more potent than cisplatin in eliminating of SiHa cells via apoptosis with downregulation of Bcl-2 protein. Toxicology in Vitro, v. 25, n. 7, p. 1392-1398, 2011.

PARENTE, L. M., PAULA, J. R., COSTA, E. A., SILVEIRA, N. A. Calendula Officinalis: características, propriedades, químicas e terapêuticas. Arquivos de Ciências da Saúde da Unipar, v. 6, n. 2, 2002.

PARKIN, D.M., BRAY, F. The burden of HPV-related cancers. Vaccine. v. 24, n. 3, p. S11-S25, 2006.

RABELO, A.M.S., BRETAS, M.L., GUILLO, L.A. Ensaio da citotoxicidade de curcumina. Universidade Federal de Goiás. Departamento de Ciências Fisiológicas - Instituto de Ciências Biológicas, Goiânia, 2002.

ROMAGNOLI, C., BRUNI, R., ANDREOTTI, E., RAI, M. K. Chemical characterization and antifungal activity of essential oil of capitula from wild Indian Tagetes patula L. Protoplasma, v. 255, n. 1, p. 57-65, 2005.

SANDERS, N. A., SIMPSON, F., THOMPSON, E. W., HILL, M. M., ENDO-MUNOS, L., LEGGATT, G., GUMINSKI, A. Role of intratumoural heterogeneity in cancer drug resistance: molecular and clinical perspectives. EMBO molecular medicine, v. 4, n. 8, p. 675-684, 2012. 
SCHIAVON, D. A. B., SCHUCH, L. F. D., FACCIN, A., GONÇALVES, C. L., VIEIRA, V. S. C., GONÇALVES, H. P. Revisão sistemática de Tagetes minuta $L$. (Asteraceae): uso popular, composição química e atividade biológica. Science and Animal Health, v. 3, p. 192-208, 2015.

SILVA, S. M. Pitanga. Revista Brasileira de Fruticultura, v. 28, n. 1, 2006.

SILVA, A. A., ANDRADE, L. H. C. Utilização de espécies de Asteraceae por comunidades rurais do nordeste do Brasil: relatos em Camocim de São Félix, Pernambuco. Biotemas, v. 26, n.2, p. 93-104, 2013.

SOUTTER, W. P., SASIENI, P., PANOSKALTSIS, T. Long-term risk of invasive cervical cancer after treatment of squamous cervical intraepithelial neoplasia. International Journal of Cancer, v. 118, n. 8, p. 2048-2055, 2006.

STEFANELLO, M. É. A., PASCOAL, A. C. R. F., SALVADOR, M. J. Essential Oils from Neotropical Myrtaceae: Chemical Diversity and Biological Properties. Chemistry e Biodiversity, v. 8, n. 1, p. 73 - 94, 2011.

TENEN, D.G. Disruption of differentiation in human cancer: AML shows the way. Nature Reviews, v. 3, p. 89-101, 2003.

VICTORIA, F.N. Estudo do potencial farmacológico do óleo essencial das folhas da Eugenia uniflora L. Tese de doutorado. PPG em Ciência e Tecnologia Agroindustrial. Universidade Federal de Pelotas, 2013.

WALBOOMERS, J. M., JACOBS, M. V., MANOS, M. M., BOSCH, F. X., KUMMERS, J. A., SHAH, K. V., SNIJDERS, P. J., PETO, J., MEIJER, C. J., MUNOZ, N. Human papillomavirus is a necessary cause of invasive cervical cancer worldwide. The Journal of Pathology, v.189, n. 1, p. 12-19, 1999.

YUAN, C., WANG, P., LAI, C., TSU, E., YEN, M., NG, H. Recurrence and survival analyses of 1,115 cervical cancer patients treated with radical hysterectomy. Gynecologic and Obstetric Investigation, v. 47, n. 2, p.127-132,1999. 